\title{
Lifetime Optimization for Partial Coverage in Heterogeneous Sensor Networks
}

\author{
Jean-Claude Charr, Karine Deschinkel, Rania Haj Mansour, Mourad Hakem* \\ University of Bourgogne Franche-Comte \\ FEMTO-ST Institute, UMR 6174 CNRS \\ Belfort, France \\ firstname.lastname@univ-fcomte.fr
}

\begin{abstract}
In this work, we investigate the problem of lifetime optimization for partial coverage in heterogeneous sensor networks. This problem which is NP-Hard in its general form is known under the name of $\alpha$-coverage, where $\alpha$ refers to a prescribed level of coverage threshold that we need to maintain. Sleep-Awake scheduling which turns sensors to On and Off, is the common and the well known technique that has been heavily studied in the literature to deal with energy management under coverage constraint. The question is how to orchestrate the clustering of the sensor nodes into disjoint or non-disjoint covers, and to schedule these covers, so that the total network's lifetime is maximized. Unlike earlier works, we consider both global (whole targets) resp. local (individual target) monitoring thresholds to improve the coverage quality rather than dealing with a single global leveling threshold as in the literature. In addition, instead of employing a default covers' activation which may lead to the starvation phenomenon, where targets may remain uncovered for a long time period, we provide a clairvoyant scheduling for the obtained covers to ensure fair smoothing for the cumulated target's uncovered time periods during the network's service. First, a novel mathematical Binary Integer Linear Programming (BILP) is proposed to solve the $\alpha$-coverage problem to optimality. Then, provable guarantees of the upper bound for the number of partial cover sets are given. Next, we formulate the covers' planning as a $p$-dispersion problem and due to the NP-Completeness of the former, an efficient Genetic Algorithm (GA) based approach is designed to achieve efficient covers' scheduling with minimal execution time complexity. Finally, a series of experiments are conducted and several QoS metrics are evaluated to show the usefulness of our proposals.
\end{abstract}

Index Terms - sensor networks; lifetime optimization; partial coverage; integer linear programming; p-dispersion; genetic algorithms

\section{INTRODUCTION}

With the emergence of IoT, wireless sensor networks (WSN) are widely used for monitoring in diverse fields of applications such as tracking, home security, tactical surveillance, health care, and so on. They are made up of many low powered and small device nodes which collaborate with each other to monitor, collect, process, and forward the sensed information using wireless communications. Nevertheless, WSNs present a number of shortcomings that may have an adverse effect on the gathered data at the sink level, leading to non reliable diagnostics of the monitored targets. Consequently, to improve the network's QoS, two main critical and related issues,

\footnotetext{
${ }^{*}$ The authors are displayed in alphabetic order
}

namely the energy consumption and target coverage, need to be considered.

While some very sensitive applications require the complete coverage of all the targets during the whole lifetime of the network, others can bear less strict monitoring. Depending on the nature and the sensitivity of the monitored targets, partial coverage, where some targets may remain uncovered for a limited time period, could be tolerated in order to prolong the network's lifetime. For instance, since the probability of a forest fire occurring in the rainy season is significantly lower than in the dry season, monitoring at each time period a few random regions in the forest could be sufficient to prevent the forest from taking fire. This partial coverage would also lead to activating at each time period a smaller number of sensors than in full-coverage which would drastically reduce the sensors' energy consumption and increase the network's overall lifetime [1]. Pollution monitoring systems can also make do with partial coverage of the monitored area. Excluding, at each time period, some random regions and computing the average pollution level using a percentage of the measurements, would not practically affect the final results [2].

Although, both energy saving and coverage requirement have been studied in the literature, to the best of our knowledge, none of the existing research works has considered, at the same time, both global (whole targets) and local (individual target) monitoring level threshold constraints nor the starvation phenomenon that may occur if the obtained cover sets are not scheduled in a suitable way. Going further, it is usually assumed that the lifetime of the partial coverage must be at least as well as the achieved one in the case of complete coverage. We strongly conjecture that this assumption is a weaker condition and it is far from being sufficient to provide reliable targets' coverage.

In this paper, we bring answers to the aforementioned shortcomings of the previous works in the literature. We target the case of Non-Disjoint Set Covers (NDSC) problem in which sensors can participate to more than one cover set and can interchange between idle and working modes. We consider heterogeneous networks where the initial energy levels of nodes' batteries are different. The aim of this paper is to deal with energy saving subject to a prescribed leveling threshold of the coverage quality that we have to ensure during the network's activity. To this end, two main and distinct 
optimization problems are investigated: i) the construction of the $\alpha$-cover sets (by the exact resolution of a binary integer linear program) and ii) the planning of the generated $\alpha$-cover sets (in which order they should be activated successively?). The output of the former is the input of latter.

In the following, we summarize the contributions and the novelties of of the presented study:

- A new mathematical Binary Integer Linear Programming (BILP) formulation is proposed to solve to optimality the Heterogeneous Non-Disjoint Partial Set Cover (HNDPSC) problem with fixed activation time periods.

- We provide necessary and sufficient global and local coverage constraints to achieve an efficient trade-off between energy and coverage performance related objectives during the network's service. The findings of our research study reveal that, when dealing with partial coverage under energy constraint, local (individual target) coverage constraint plays a crucial role on the achieved global performances of the network's monitoring activity (See Section III for more details on these constraints called resp. $\alpha$ for the global constraint and $\beta$ for the local one).

- We give provable guarantees for two upper bounds for the number of non-disjoint cover sets that can be constructed when dealing with partial coverage under fixed activation time periods in heterogeneous sensor networks. This drastically reduces the number of variables which is a key factor when solving linear and nonlinear optimization problems. That is to say that these bounds allow practical gains and enable us to solve the $\alpha$-coverage problem in a single stage in contrast with what was previously proposed in the literature.

- To avoid the starvation phenomenon, instead of considering a default activation of the resulting cover sets, we provide an efficient scheduling to fairly smooth the target's uncovered time periods during the network's lifetime. To this end, first we formulate the dispersion of uncovered time periods of a target throughout the network's lifetime as a $p$-dispersion problem. Then, we derive a generalization of the $p$-dispersion problem where the dispersion of the uncovered time periods for all the monitored targets should be optimized at the same time. Two criteria were adopted to reflect how well the uncovered time periods of all targets are balanced (See Section IV for more details on these criteria). Due to the NP-completeness of the $p$-dispersion problem and its generalization, an efficient GA was designed to achieve near optimal solutions in polynomial time complexity.

The remainder of this paper is organized as follows. In Section 2, the relevant $\alpha$-Lifetime optimization techniques that have been proposed in the literature are reviewed. In Section 3 , we present in details the new proposed BILP mathematical model for the HNDSC problem as well as the upper bound's analysis of the number of partial cover sets. Section 4 is devoted to the Cover Set Scheduling which includes the pdispersion problem formulation and the presentation of a GA to find a good scheduling of the obtained cover sets. We report in Section 5, a series of experimental results to asses the behaviour of our proposals. In particular, our approach is compared to a competing method from the literature. Finally, some concluding remarks are made in Section 6.

\section{RELATED WORK}

In the last two decades, the Maximum Network Lifetime Problem (MLP) in wireless sensor networks had considerable attention from researchers. In [2]-[7], exact methods and heuristics were proposed to either solve small instances of the problem to optimality or produce good solutions for large instances in a reasonable time. Solving the MLP consists in finding subsets of sensors that can cover all the targets for the longest possible time period. The MLP was shown to be NP-complete by a polynomial time reduction from the well known problem 3-SAT [8].

Several derived problems from the MLP were proposed to adapt it to different contexts. Some of them address coverage connectivity [9] [10], reliability [11], or consider sensors with adjustable coverage range [12] [13]. Another interesting variant of the problem, studied in [2], [4], [13] and [5], is the $\alpha$-Maximum Network Problem ( $\alpha$-MLP), in which a given portion $((1-\alpha)$ percent $)$ of the targets could be uncovered in each cover set. In [2] and [4], the authors demonstrated that in some cases, it is preferred to partially cover the targets for a longer period instead of providing full coverage for a short one. They have also provided a formulation of the problem as a linear program where the objective function is the maximization of the $\alpha$-Lifetime of the WSN. This formulation first requires the generation of all possible feasible $\alpha$-covers. For a given $\alpha \in[0,1]$, an $\alpha$-cover is a subset of the sensors that covers at least $\alpha \times|T|$ targets (where $|T|$ is the total number of targets in the monitored area). Once all the $\alpha$-covers have been generated, the resolution method have to find out how much time each $\alpha$-cover has to be activated. Therefore, the linear program's variables are the activation times of all the feasible $\alpha$-covers and its objective function is the maximization of the sum of their activation times while ensuring that the battery lifetime of each sensor is not exceeded.

Since the number of potential $\alpha$-covers increases exponentially with the number of sensors, especially for lower values of $\alpha$, the authors applied a Column Generation (CG) approach to be able to find the optimal solutions for small instances of the problem in reasonable times. The same approach was already proposed in [14] to solve the MLP. At each iteration of the CG method, a Restricted Master Problem, with only a subset of the feasible $\alpha$-covers, is solved. Then a specific optimization problem (generally called subproblem) is solved which either produces an attractive cover to be considered while solving the Master Problem in the next iteration or guarantees that the last found solution found is the optimal one. 
In [2] the subproblem was formulated as a integer linear program (ILP) and solved to optimality. In [4], the authors attempted to heuristically solve the subproblem by using a genetic meta-heuristic. In both works, an additional constraint was added to the Restricted Master problem such that each target is at least covered as in the complete coverage problem (with $\alpha=1$ ). Therefore, before solving the $\alpha$-MLP for a given instance, the complete coverage problem must be solved for the same instance in order to find out what is the minimal coverage time to respect for each target. The need to go through this preliminary step is one of the major disadvantages of this approach. The authors also proposed in the same paper a greedy approach, called $\alpha-$ greedy, to find feasible $\alpha$-covers and to initialize the Column Generation procedure. They assigned a predefined activation time to each generated $\alpha$-cover. Their heuristic iteratively constructs each $\alpha$-cover by adding to it the sensor with the highest residual energy and at the same covering the largest number of uncovered targets. In [13], another greedy algorithm for partial coverage of WSNs was proposed as well. In this work, the nodes have different sensing and communication ranges but the same amount of initial energy. The proposed algorithm guarantees the connectivity of the nodes while constructing the $\alpha$-covers. The covers sets are then successively activated during a fixed amount of time $\lambda$ such that a sensor could participate in several cover sets. However, this approach does not guarantee that each target will be sufficiently monitored over the entire lifetime of the network.

In [5], a heuristic that provides the maximum number of $\alpha$-cover sets, was presented. These cover sets were activated one by one for a fixed time period. As in [2] and [4], a minimal coverage time per target, equal to their coverage time in the complete coverage problem, was ensured. Therefore, this approach also requires the pre-calculation of the minimum coverage level for each target. The authors of this paper claim that it is possible to extend the network lifetime by wisely selecting the targets to be uncovered in each cover set. However, their approach and simulations are limited to homogeneous sensors (all the sensors have one initial energy unit) and therefore each sensor can at most be involved in two cover sets (the activation time of a cover set is fixed to 0.5 unit). Even though the network's lifetime is extended in most cases, for some instances some targets are monitored less than $20 \%$ of the network lifetime which can be potentially dangerous.

The work presented in [7] is the closest one to our study because it also proposes an exact method for solving the coverage problem in a heterogeneous wireless sensor network (sensors with non-identical amount of initial energy and power consumption). The authors of [7] present an Integer Linear Programming (ILP) mathematical model for maximizing the network lifetime. Their goal is to find out how many times each possible cover set should be activated during a fixed amount of time. Their model can be easily extended to partial coverage. More details about this technique are given in section III-D. But the major drawback of this method, as shown in our experiments in Section V-B, is that it requires two time-consuming preliminary steps in order to generate all the possible cover sets.

The authors of [2], [4], [5], [7], [13] proposed exact or heuristic methods for solving the $\alpha$-MLP but none of them took into account the fact that the coverage period for each target may be too short when compared to the total lifetime of the network. Therefore, in our approach, although the network lifetime is partially reduced, we guarantee that each target will be covered for a minimum percentage of the network lifetime, which is more appropriate to real-life applications requirements. In addition, to the best of our knowledge, no method is proposed in the literature for scheduling $\alpha$ - cover sets once they have been generated. This is why we provide a judicious way to schedule the $\alpha$-cover sets in order to avoid excessively long periods of time during which some targets are not monitored.

\section{PROBlem Formulation}

In this section, we define more formally the $\alpha$ - Maximum Lifetime problem and in order to solve it, it is modeled as a Binary Integer Linear Programming (BILP) problem.

\section{A. Notations}

Before getting in details, we first define some notations that will be used throughout the paper.

- $\mathrm{n}$ : Number of sensors

- $\mathrm{m}$ : Number of targets

- S : Set of sensors $=\left\{s_{1}, \ldots, s_{n}\right\}$

- $\mathrm{T}:$ Set of targets $=\left\{t_{1}, \ldots, t_{m}\right\}$

- $E_{i}$ : Available time units for sensor $s_{i}$

- $T_{i}$ : Set of targets covered by the sensor $s_{i}$

- $S_{j}$ : Set of sensors covering the target $t_{j}$

- $C_{k}: \alpha$ - cover set $k$

- $d$ : Fixed activation time of a partial cover set $C_{k}$.

We assume that $n$ heterogeneous sensors are deployed to monitor $m$ targets. Sensors might have heterogeneous initial battery power and power consumption. After deployment, each sensor $s_{i}$ has a battery level $B_{i}$ and an energy consumption of $e_{i}$ per unit of time. For each sensor, $E_{i}$ represents the number of time units during which it can be activated continuously such that $E_{i}=\frac{B_{i}}{e_{i}}$. In the rest of the paper, we will indifferently use the term energy or time units to refer to the quantity $E_{i}$ for sensor $s_{i}$. We consider a classic coverage model which consists of saying that a target $t_{j}$ is covered by a sensor $s_{i}$ if and only if the distance (Euclidean distance) between $t_{j}$ and $s_{i}$ is less than the coverage radius of the sensor $s_{i}$. The matrix $\Delta$ is defined with the Boolean coefficients $\delta_{i j}$ such that $\delta_{i j}$ is equal to 1 if the target $t_{j}$ is covered by the sensor $s_{i}$ and 0 otherwise.

In order to optimize the lifetime of the network, redundant sensors are scheduled to sleep when their targets are being monitored by other active sensors. This leads to the construction of cover sets consisting of sensors that cover the targets for a given time period. Then, these cover sets are activated one after the other. In the partial coverage context, for a given 
$\alpha \in(0,1], C_{k} \subseteq S$ is an $\alpha$-cover set if its sensors cover at least $T_{\alpha}=\lfloor\alpha \times m\rfloor$ targets. The $\alpha$-cover sets can be non-disjoint which means a sensor can participate to more than one cover set if it has enough energy. In this work, we assume that all the cover sets have the same activation time $d$. Therefore, improving the lifetime of the network amounts to maximizing the number of constructed $\alpha$-cover sets. As in other models [5] [13] in the literature, the activation time is a fixed parameter. Its value should be long enough to hide the system control overhead and short enough to minimize the negative effects in case of node failures. In this paper, to concentrate our efforts on the introduction of new types of constraints to prevent some targets from being uncovered during a long time period in the case of partial coverage, we have assumed that the duration of the activation time is fixed. Concerning the choice of the value of the fixed activation time $d$, it is correlated to the type of the considered application and the sensors initial energies.

When the coverage is partial, all the targets do not have the same coverage rate which can lead to very poor coverage of some individual targets. Therefore, it is appropriate to add additional constraints to ensure for each target a minimum coverage rate over the total lifetime of the network. We introduce a new parameter $\beta$ which defines the minimal ratio between the time of coverage of one target and the network lifetime. We denote this parameter $\beta$ as a "Target Monitoring Ratio" applied to each target whereas the coverage ratio $\alpha$ is applied to each cover set. Therefore, the new objective of the $\alpha$ - Maximum Lifetime Problem is to form as many $\alpha$-cover sets as possible while meeting coverage and energy constraints.

\section{B. BILP: model formulation}

The search for the optimal solution to the $\alpha-M L P$, can be formulated as a Binary integer linear programming (BILP) problem. Since all the cover sets have a fixed activation time, the goal of the BILP is to construct the maximum number of $\alpha$-cover sets. The upper bound of the possible number of $\alpha$-cover sets for a given instance can be denoted by $K$ and its calculation is discussed in section III-C.

The variables used to define the problem are the following:

- Binary variables $x_{i, k}, \forall i \in \llbracket 1, n \rrbracket$ and $\forall k \in \llbracket 1, K \rrbracket$; $x_{i, k}=1$ means that the sensor $s_{i}$ is active in the cover set $C_{k}$.

- Binary variables $y_{j, k}, \forall j \in \llbracket 1, m \rrbracket$ and $\forall k \in \llbracket 1, K \rrbracket$; $y_{j, k}=1$ means that the target $t_{j}$ is covered by the cover set $C_{k}$.

- Binary variables $\mathbf{z}_{\mathbf{k}}, \forall k \in \llbracket 1, K \rrbracket ; z_{k}=1$ means that $C_{k}$ is an $\alpha$-cover set.

1) Objective: The objective is to maximize the number of $\alpha$-cover sets.

$$
\operatorname{Max} \sum_{k=1}^{K} z_{k}
$$

2) Global coverage constraints: If the sensor $s_{i}$ is active in the cover set $C_{k}$, the set of targets $\left(T_{i}\right)$ that it monitors will be covered in the cover set $C_{k}$. A target $t_{j}$ is covered if there is at least one sensor $s_{i} \in S_{j}$ that monitors it in the set $C_{k}$. This is mathematically formulated by the following two types of constraints:

$$
\begin{gathered}
y_{j, k} \geq x_{i, k} \forall j \in \llbracket 1, m \rrbracket, \forall k \in \llbracket 1, K \rrbracket, \forall i \in S_{j} \\
\sum_{i \in S_{j}} x_{i, k} \geq y_{j, k} \forall j \in \llbracket 1, m \rrbracket, \forall k \in \llbracket 1, K \rrbracket
\end{gathered}
$$

Constraint (2) forces the variable $y_{j, k}$ to be equal to 1 if one sensor of $S_{j}$ is activated in the $\alpha$-cover. Constraint (3) allows the variable $y_{j, k}$ to be equal to 1 only if at least one of the sensors monitoring it is active in the cover set $C_{k}$.

The following constraints impose that at least $T_{\alpha}$ targets are covered in each $\alpha$-cover set :

$$
\sum_{j \in T} y_{j, k} \geq T_{\alpha} \times z_{k} \forall k \in \llbracket 1, K \rrbracket
$$

3) Target's coverage constraints: As explained above, our model includes a new type of constraints that limits the network lifetime according to the parameter $\beta$ (Target Monitoring Ratio) such that the total coverage time of each target is greater than or equal to $\beta$ percent of the network lifetime. Moreover, in some applications such as forest fires, it is necessary to monitor the coverage of the targets that have been affected by the fires. These targets must have a higher monitoring ratio than the others and then each target $j$ has its own monitoring ratio $\beta_{j}$. This constraint is called in this paper $\beta$ constraint and it can be expressed as follows :

$$
\sum_{k=1}^{K} y_{j, k} \geq \beta_{j} \times \sum_{k=1}^{K} z_{k} \forall j \in \llbracket 1, m \rrbracket
$$

$\sum_{k \in K} y_{j, k}$ represents the number of $\alpha$-cover sets which cover the same target $t_{j}$, and $\sum_{k \in K} z_{k}$ is the total number of generated $\alpha$-cover sets.

The $\beta$ constraint differs from those usually proposed in the literature for partial coverage. Authors of [2], [4] and [5] have proposed the $w_{\text {min }}$ constraint which imposes that each target must be covered at least as well as the achieved one in the case of complete coverage. This kind of constraint requires the resolution of the model with $\alpha=1$ beforehand to provide a common minimum coverage bound $w_{\min }$ for the whole targets and it can be expressed as follows :

$$
\sum_{k=1}^{K} d \times y_{j, k} \geq w_{\min } \forall j \in \llbracket 1, m \rrbracket
$$

4) Energy constraints: In the non-disjoint case, a sensor might belong to several $\alpha$-cover sets if it has enough energy. The following constraint ensures that the total energy consumed by a sensor does not exceed its initial energy:

$$
\sum_{k=1}^{K} d \times x_{i, k} \leq E_{i} \forall i \in \llbracket 1, n \rrbracket
$$


5) Additional constraints: To make the model consistent and ensure that the sets that do not respect the $\alpha$-cover set conditions (i.e $z_{k}$ is equal to 0), are empty, the following constraint has been added to the model:

$$
\sum_{i \in S} x_{i, k} \leq n \times z_{k} \forall k \in \llbracket 1, K \rrbracket
$$

This constraint forces the variables $x_{i, k}$ to be equal to zero if $C_{k}$ is not an $\alpha$-cover set.

6) Optional constraints: By construction, the total coverage time of a target cannot exceed the total time of the sensors capable of monitoring it. This constraint can be formulated as follows:

$$
\sum_{k=1}^{K} d \times y_{j, k} \leq \sum_{i \in S_{j}} E_{i} \forall j \in \llbracket 1, m \rrbracket
$$

This constraint is not mandatory but we have noticed that by adding this extra constraint, the resolution time of the Branchand Bound method for the BILP is significantly reduced. This constraint can be seen as a cutting plane in the resolution process.

Considering cover sets of fixed duration $d$, a Coverage Ratio $\alpha$ and a Target Monitoring Ratio $\beta$, a new mathematical formulation of the $\alpha$-Maximum Lifetime Problem can be given as follows:

$$
\begin{cases}\max \sum_{k=1}^{K} z_{k} & \\ \text { subject to : } & \forall j \in \llbracket 1, m \rrbracket, \forall i \in S_{j}, \\ y_{j, k} \geq x_{i, k} & \forall k \in \llbracket 1, K \rrbracket \\ & \forall j \in \llbracket 1, m \rrbracket, \forall k \in \llbracket 1, K \rrbracket \\ \sum_{i \in S_{j}} x_{i, k} \geq y_{j, k} & \forall k \in \llbracket 1, K \rrbracket \\ \sum_{j \in T} y_{j, k} \geq T_{\alpha} \times z_{k} & \forall i \in \llbracket 1, n \rrbracket \\ \sum_{k=1}^{K} d \times x_{i, k} \leq E_{i} & \forall j \in \llbracket 1, m \rrbracket \\ \sum_{k=1}^{K} y_{j, k} \geq \beta \times \sum_{k=1}^{K} z_{k} & \forall k \in \llbracket 1, K \rrbracket \\ \sum_{i \in S} x_{i, k} \leq n \times z_{k} & \forall j \in \llbracket 1, m \rrbracket \\ \sum_{k=1}^{K} d \times y_{j, k} \leq \sum_{i \in S_{j}} E_{i} & \end{cases}
$$

It's worthwhile to note that the number of variables is $(n+$ $m+1) \times K$. The number of constraints is bounded by $m n K+$ $m K+2 K+n+2 m$. Consequently, it is not surprising that the resolution of this linear program with binary variables becomes impracticable for large optimization problems.

\section{$C$. The upper bound of the number of $\alpha$-cover sets, $K$}

In this section, we give two upper bounds of the number of $\alpha$-cover sets for the problem of partial coverage in WSN where each $\alpha$ - cover set is activated during a fixed time period (called slot) of $d$ time units. First, we start by computing the general upper bound $K$, next we derive a tighter one $K^{\prime} \leq K$ and prove its attainability. Finally, we shall express a bound on the maximum number of $\alpha$-cover sets in the special case where a Target Minimum Ratio, $\beta$, is required for each target.

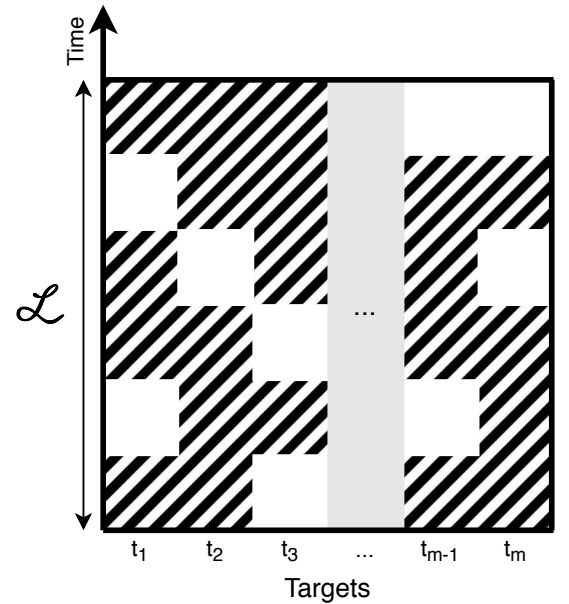

Fig. 1: The cumulated idle and coverage time slots of the $m$ targets.

Proposition 1. The number of cover sets for the $\alpha$-coverage problem is upperbounded by

$$
K=\left\lfloor\frac{\sum_{\ell=1}^{m} \sum_{i \in S_{\ell}} E_{i}}{\alpha \times m \times d}\right\rfloor
$$

Proof. Consider the clustering illustrated in Figure 1. Since we deal with partial coverage, at any time step in the network's activity, some targets are covered and some others are not. Let $\mathcal{I}$ and $\mathcal{C}$ be the cumulated Idle time slots, resp. the cumulated Coverage time slots of the $m$ targets throughout the lifespan of the network.

Intuitively, we have:

$$
\mathcal{I}+\mathcal{C}=\mathcal{L} \times m
$$

where $\mathcal{L}$ is the Upper Bound of the achieved network's lifetime. Since, the cover's duration time is the same for all the constructed covers, we obtain:

$$
\mathcal{I}+\mathcal{C}=K \times d \times m
$$

Moreover, we can observe that:

$$
\mathcal{C}=K \times d \times m \times \alpha
$$

In this way we deduce:

$$
K=\left\lfloor\frac{\mathcal{C}}{\alpha \times m \times d}\right\rfloor=\left\lfloor\frac{\sum_{\ell=1}^{m} \sum_{i \in S_{\ell}} E_{i}}{\alpha \times m \times d}\right\rfloor
$$

Proposition 2. Let $\Delta>0$ be the cumulated residual energy that cannot be used to form new covers, then the Upper Bounded $K$ can be reduced down to

$$
K^{\prime}=K-\varepsilon
$$

where, $\varepsilon$ is within

$$
\left\lfloor\frac{\Delta}{\alpha \times m \times d}\right\rfloor
$$


Proof. We need to prove that $K^{\prime} \leq K$ holds. According to the Greedy-Procedure's policy (see Algorithm 1), a cover set is built if and only if it remains enough energy that could be assigned to $\alpha \times m$ targets. Let $\lambda$ be the remaining cumulated energy in the time slot at the $i$ 'th iteration, $1 \leq i \leq K$. Then, the number of constructed cover sets at the time step $i$ during the clustering process is:

$$
i-1+\left\lfloor\frac{\lambda}{\alpha \times m \times d}\right\rfloor \leq K
$$

Now, we consider the worst case where all the computed covers are holding the needed value of $\alpha \times m$ targets except for the last one which cannot be retained owing to the condition pointed above. In this configuration, the whole amount of the residual energy, denoted as $\Delta$, that will no longer be usable before reaching the final number of cover sets will be decreased from the global energy of the network. Thus,

$$
\begin{aligned}
& K^{\prime}=\left\lfloor\frac{\sum_{\ell=1}^{m} \sum_{i \in S_{\ell}} E_{i}-\Delta}{\alpha \times m \times d}\right\rfloor \\
& \leq\left\lfloor\frac{\sum_{\ell=1}^{m} \sum_{i \in S_{\ell}} E_{i}}{\alpha \times m \times d}\right\rfloor+\left\lfloor\frac{-\Delta}{\alpha \times m \times d}\right\rfloor+1 \\
& =\left\lfloor\frac{\sum_{\ell=1}^{m} \sum_{i \in S_{\ell}} E_{i}}{\alpha \times m \times d}\right\rfloor-\left\lceil\frac{\Delta}{\alpha \times m \times d}\right\rceil+1 \\
& =K-\left\lceil\frac{\Delta}{\alpha \times m \times d}\right\rceil+1 \\
& \Longrightarrow K^{\prime} \leq K+1-\left\lceil\frac{\Delta}{\alpha \times m \times d}\right\rceil
\end{aligned}
$$

We have two scenarios:

1) $0<\Delta \leq \alpha \times m \times d \Longrightarrow K^{\prime}=K$

2) $\Delta>\alpha \times m \times d \Longrightarrow K^{\prime}<K$

$$
\text { (1) and }(2) \Longrightarrow K^{\prime} \leq K
$$

Hence a result,

$$
K^{\prime}=K-\varepsilon \wedge \varepsilon \leq\left\lfloor\frac{\Delta}{\alpha \times m \times d}\right\rfloor
$$

Proposition 3. The bound $K^{\prime}$ is attainable.

Proof. To see that this bound is really attainable, consider a network of two sensors $(n=2)$ which are deployed to cover two targets $(m=2)$. Assume a one-to-one scenario where each sensor is a assigned to a separate target. Let $\alpha=0.5$, $E_{1}=E_{2}=1$ and $T_{1} \bigcap T_{2}=\phi$. It's straightforward to check that the achieved lifetime is $L=2$ with $K^{\prime}=2$ covers. This result is optimal and cannot be improved.

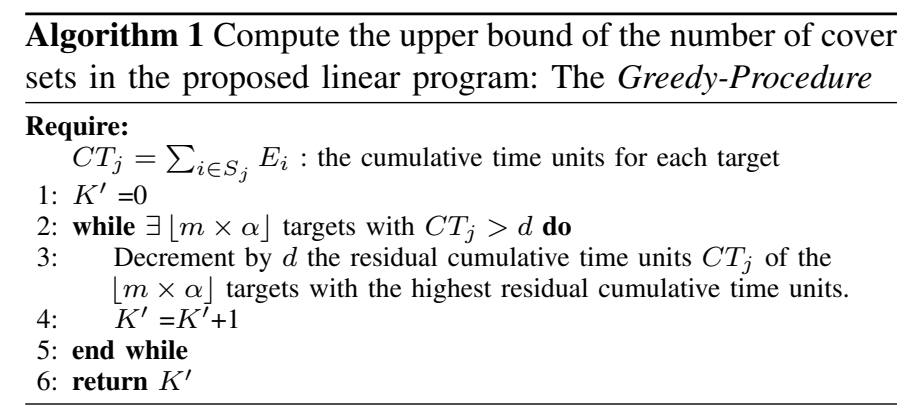

In our BILP formulation, when the Constraint (5) with the Target Minimum Ratio $\beta_{j}$ is applied, the maximum number of non-disjoint $\alpha$-cover sets of a fixed activation time period $d$, is bounded by the least covered target and $\beta_{j}$. Thus, this upper bound can be computed as the following:

$$
K=\min _{j \in T}\left\lfloor\frac{\sum_{i \in S_{j}} E_{i}}{\beta_{j} \times d}\right\rfloor
$$

For the sake of comparison, we present in the following section, the description of an existing network's lifetime optimization approach introduced in [7] which is, as far as we know, the closest work to the one addressed in this paper.

\section{An existing Integer Linear Formulation}

In this section, we discuss a mathematical formulation for the Maximal Lifetime Problem in WSN designed in [7] and we present an adaptation of this method to solve $\alpha$-MLP. In this way, we will be able to compare this approach to the one proposed in this paper. To solve the MLP problem, the authors in [7] proposed a method using the three following steps :

1) Construct all possible cover sets (at most $2^{n}-1$ where $n$ is the number of sensors). Retain only the cover sets where the coverage conditions are satisfied (all targets are covered in the case of complete coverage, $\lfloor\alpha * m\rfloor$ targets are covered in the case of partial coverage). You get $L^{\prime}$ cover sets said valid.

2) Among the valid $L^{\prime}$ cover sets, retain those which are elementary (where there are no superfluous sensors) and thus with a smaller number of sensors. We get $L$ valid and elementary cover sets. Construct the matrix $A$ of binary coefficient $a_{i, l}$ which is equal to 1 if the sensor $i$ is in the cover set $C_{l}, 0$ otherwise.

3) Write the associated Integer Linear Program and solve it.

Let $u_{l}$ be the number of times the cover set $C_{l}$ is activated during a fixed activation time $d$. The mathematical model, designed by the authors of [7], can be formulated with the notation used in this paper as the following Integer Linear Program (ILP).

$$
\left\{\begin{array}{l}
\max \sum_{l=1}^{L} d \times u_{l} \\
\text { subject to : } \\
\sum_{l=1}^{L} d \times a_{i, l} \times u_{l} \leq E_{i} \quad \forall i \in \llbracket 1, n \rrbracket
\end{array}\right.
$$

The objective function expresses the network lifetime. As constraint (7), the constraints in this formulation guarantee that 
the total consumed energy by a sensor cannot exceed its initial reserve of energy (here expressed as a number of available time units $E_{i}$ for a sensor $i$ ). To introduce the $\beta$ constraint in this model, it is necessary to build the matrix $B$ where the binary coefficient $b_{j, l}$ is equal to 1 if the target $j$ is monitored in the cover set $C_{l}, 0$ otherwise. The $\beta$ constraint for this model can be formulated as the following:

$$
\sum_{l=1}^{L} b_{j, l} \times u_{l} \geq \beta_{j} \sum_{l=1}^{L} u_{l} \quad \forall j \in \llbracket 1, m \rrbracket
$$

Although this formulation seems to be simple as it involves only one type of variables and two types of constraints, its construction process is composed of two preliminary complex steps which are very time-consuming. We have called this method the 3-steps method to distinguish it from our approach (called all-in-one method) for which the construction of the covers sets and the computation of their activation times are performed in a single model. In part V, our approach is compared to the 3-steps method and the results show that our mathematical formulation outperforms the latter.

\section{Cover Sets Scheduling Problem}

The optimal solution obtained from the BILP consists of $K_{\text {opt }} \alpha$-cover sets that have a fixed activation time period $d$. These cover sets should be activated successively to cover the targets during the lifetime of the WSN. In the case of partial coverage, a target might be covered in a non continuous mode.

Let $\Theta$ be the coverage binary matrix for a given solution such that $\theta_{j, k}$ is equal to 1 if target $t_{j}$ is covered in the cover set $C_{k}$ and 0 otherwise, see matrix (14).

$$
\Theta=\left(\begin{array}{cccc}
\theta_{1,1} & \theta_{1,2} & \cdots & \theta_{1, K_{o p t}} \\
\theta_{2,1} & \theta_{2,2} & \cdots & \theta_{2, K_{o p t}} \\
\vdots & \vdots & \ddots & \vdots \\
\theta_{m, 1} & \theta_{m, 2} & \cdots & \theta_{m, K_{o p t}}
\end{array}\right)
$$

In some cases, when the cover sets are not properly scheduled, we may have situations where targets remain continuously uncovered during many successive cover sets. For example, in matrix (15), target 1 is not covered for three consecutive periods.

$$
\begin{gathered}
C_{1} \\
t_{1} \\
t_{2} \\
t_{3}
\end{gathered}\left(\begin{array}{cccccccccc}
1 & 1 & C_{3} & C_{4} & C_{5} & C_{6} & C_{7} & C_{8} & C_{9} & C_{10} \\
1 & 0 & 0 & 0 & 0 & 0 & 1 & 1 & 1 & 1 \\
0 & 1 & 1 & 1 & 1 & 1 & 1 & 1 & 1 & 1 \\
& & & & & 1 & 0 & 1 & 1 & 1
\end{array}\right)
$$

To avoid this issue which can be viewed as a starvation phenomenon, we provide in this section a meta-heuristic that searches for a good approximation of the most favourable scheduling of the obtained cover sets. For each target and as much as possible, the new scheduling should smooth fairly, during the whole lifetime of the network, the periods where a target is not covered. In other words, the new order should disperse, for every target, the zeros in the coverage matrix, $\Theta$.

As was mentioned in the introduction section, to measure the dispersion rate of the uncovered periods for a given covers' schedule, we use two key criteria, namely: the p-dispersion and the coefficient of variation criteria.

\section{A. The first criterion: Measure of dispersion (p-dispersion)}

Dispersing elements in a set has been already tackled in the literature and it is called the p-dispersion problem. Unfortunately, this problem is known to be NP-hard [15] in the general case and heuristics are required to achieve sub-optimal solutions but in polynomial time complexity.

\section{Definition IV.1. p-dispersion Problem}

Given $p$ elements and a set of $n$ locations where $p<n$, the objective of this problem is to select $\mathrm{p}$ locations where the $\mathrm{p}$ elements would be as dispersed as possible which amounts to maximizing the minimum distance (MAX-MIN) between any pair of the $p$ elements [16] [17] [18].

Let $N$ and $U$ be respectively the set of candidate locations (of size $n$ ) and the solution vector (of size $p$ ). Considering a metric space where the distance between two elements $u_{i}$ and $u_{j}$ is denoted by $\operatorname{dis}\left(u_{i}, u_{j}\right)$ and the identity of indiscernible, symmetry and triangle inequality properties are satisfied, the discrete p-dispersion problem can be stated as the following:

$$
\left\{\begin{array}{l}
\max (f(U)) \\
\text { Subject to: } \\
\quad f(U)=\min \left(\operatorname{dis}\left(u_{i}, u_{j}\right): 1 \leq i<j \leq p\right) \\
\quad U \subset N,|U|=p
\end{array}\right.
$$

In our case, for a given target $t_{i}$, the indexes of the vector $\left(\theta_{i, 1}, \cdots, \theta_{i, K_{o p t}}\right)$ are the locations and the $p$ elements to disperse in these locations are the coefficients of that vector that are equal to 0 . The distance between two elements is equal to the absolute value of the difference between their indexes minus $1, \operatorname{dis}\left(\theta_{i, x}, \theta_{i, y}\right)=|x-y|-1$, with $x \neq y$. For example, if $\theta_{i}=(1,1,0,1,1,0,1,1,1,0,1)$, $\operatorname{dis}\left(\theta_{i, 3}, \theta_{i, 6}\right)=2, \operatorname{dis}\left(\theta_{i, 5}, \theta_{i, 9}\right)=3$ and the minimum distance between the coefficients equal to $0, \min (d i s)$, is equal to 2. To well disperse the uncovered periods of a target, the minimum distance should be maximized. Moreover, in order to not always have the first and last periods uncovered, the extremities of the vector could be assumed as uncovered periods and thus, in the last example, the minimum distance between the coefficients equal to 0 or the extremities, is equal to 1 . The best dispersion of the uncovered periods in this example is the following: $\theta_{i}=(1,1,0,1,1,0,1,1,0,1,1)$ where $\min ($ dis $)=2$.

The p-dispersion problem should be applied to each target in order to disperse, along the whole lifetime of the network, the periods where a target is not covered. A cover set in the solution, obtained by solving the BILP optimization problem, represents, for a given activation time period, which target is covered or not. Then modifying the cover's schedule to disperse the uncovered time periods of one target might jeopardize the dispersion rate of the other targets' uncovered time periods. Therefore, in this case the objective should be 
maximizing the minimum of the minimum distances for each target. The problem can be stated as the following:

$$
\left\{\begin{array}{l}
\max \left(\min _{l=1}^{m}\left(f\left(U_{l}\right)\right)\right) \\
\text { Subject to: } \\
f\left(U_{l}\right)=\min \left(\operatorname{dis}\left(u_{i}, u_{j}\right): 1 \leq i<j \leq p\right) \\
N=\theta_{l} \\
U_{l}=\left\{\theta_{l, j} / \theta_{l, j}=0, j=1, \cdots, K_{\text {opt }}\right\}
\end{array}\right.
$$

From this formulation, it can be seen that the minimum of the minimum distances between the uncovered periods for each target in the coverage matrix (15), is equal to 0 . If the same covers are scheduled as in the coverage matrix (18), the minimum of the minimum distances is equal to 1 .

$C_{9}$
$t_{1}$
$t_{2}$
$t_{3}$$\left(\begin{array}{cccccccccc}1 & 1 & C_{8} & C_{4} & C_{1} & C_{6} & C_{7} & C_{3} & C_{5} & C_{10} \\ 1 & 0 & 1 & 0 & 1 & 0 & 1 & 1 & 0 & 1 \\ 1 & 1 & 1 & 1 & 0 & 1 & 0 & 1 & 1 & 1\end{array}\right)$

The same covers can also be ordered as in the coverage matrix (19) which has the minimum of the minimum distances also equal to 1 . To differentiate between two solutions with same minimum of the minimum distances, as in the previous two coverage matrices, another criterion must be used. In the next subsection, the coefficient of variation criterion is presented.

$C_{9}$
$t_{1}$
$t_{2}$
$t_{3}$$\left(\begin{array}{cccccccccc}1 & 0 & C_{8} & C_{4} & C_{1} & C_{2} & C_{7} & C_{3} & C_{5} & C_{10} \\ 1 & 1 & 1 & 0 & 1 & 1 & 1 & 1 & 0 & 1 \\ 1 & 1 & 1 & 1 & 0 & 1 & 0 & 1 & 1 & 1\end{array}\right)$

\section{B. The second criterion: coefficient of variation}

Two distinct solutions having the same minimum of the minimum distances (first criterion) does not imply that both solutions have the same dispersion rate for the uncovered periods. Moreover, solutions giving the same minimum of the minimum distances is very common especially when the ratio of the maximum number of uncovered periods per target to the number of periods $\left(\max _{i=0}^{m}\left(p_{i}\right) / K_{\text {opt }}\right)$ is high. The number of uncovered periods per target, $p_{i}$, depends on $\beta$. To differentiate such solutions, we propose to use the average of the coefficients of variation (CV) criterion. Indeed, if the uncovered periods of one target are well dispersed, the distances between its successive uncovered periods should be very close to the average of these distances. Therefore, if the $\mathrm{CV}$ of a target is low, these distances are very close to their average and the uncovered periods are well dispersed. The coefficient of variation was used instead of the standard deviation, because the number of uncovered periods might be different from one target to the other. The relative value of the $\mathrm{CV}$ allows its comparison to the CVs of other targets.
The CV of the distances between the successive uncovered periods of a target, $t$, with the coverage vector $\theta_{t}=$ $\left(\theta_{t, 1}, \cdots, \theta_{t, K_{\text {opt }}}\right)$ can be computed as follows:

Let $I=\left(i_{1}, \cdots, i_{p}\right)$ be an ordered set containing the indexes of the coefficients equal to 0 in $\theta_{t}$ and $|I|=p_{t}$.

Let $D=\left(d_{0}, \cdots, d_{p}\right)$ be the set of distances between the coefficients equal to 0 in $\theta_{t} \cdot d_{0}=i_{1}-1$ is the distance between the left extremity and the first coefficient equal to 0 . For $k=1, \cdots, p-1, d_{k}$ is the distance between the coefficients of indexes $i_{k}$ and $i_{k+1} . d_{p}$ is the distance between the last coefficient which is equal to 0 and the right extremity. $\mathrm{CV}$ is equal to the standard deviation to the mean of the vector D.

For example, for $\theta_{t}=(1,1,0,1,1,0,1,1,1,0,1)$, the $\mathrm{CV}$ of target $t$ can be computed as follows:

$$
\begin{aligned}
& \mu_{t}=(2+2+3+1) / 4=2 \\
& \sigma_{t}^{2}=(2-2)^{2}+(2-2)^{2}+(3-2)^{2}+(1-2)^{2}=2 \\
& C V_{t}=\sigma_{t} / \mu_{t}=\sqrt{2} / 2
\end{aligned}
$$

To consider the dispersion of uncovered periods for all the targets in a solution, the average of the CVs of all the targets is computed. When two solutions have the same value for the first criterion, the one having the lowest average CVs is considered to have more dispersed uncovered periods than the other. The scheduling 18 and 19 give the same value for the first criterion. Their respective average $\mathrm{CVs}$ are equal to 0.87 and 1.06 and therefore the first scheduling is considered to have well balanced uncovered time periods than the second one.

\section{Method of resolution: Genetic Algorithm}

Since, the cover sets scheduling problem is a hard problem and some solutions could consist of a large number of cover sets, we present in this section a genetic algorithm (GA) to find good solutions to this problem in a reasonable time and maximize the dispersion of the uncovered periods in the optimal solution obtained by the BILP. Before going into in details, we first pay a little attention on the rationale of our choice for GA metaheuristic [19] in order to tackle the second optimization problem addressed in this paper. Indeed, broadly speaking, other metaheuristics optimization algorithms may be more efficient than GA in terms of performances and convergence speed, but the metaheuristics suitability relies on the amount of knowledge and the kind of the problem that we are facing. It was shown, in the literature, that GAs are prevalent and natural candidates for ordering optimization problems like job scheduling, vehicle routing problem (VRP) or the well-known, a special case of the later, travelling salesman problem (TSP). They are often able to achieve better tradeoffs between the solution's quality and the induced computing time. Moreover, the chromosomes' representation ensures that, at each iteration step, the whole genotypic space corresponds to feasible solutions. In our study, it turns out that the second optimization problem of covers' planning, in particular the $p$ dispersion problem and its generalization for all the monitored 
targets can be seen as an ordering optimization problem. Hence the rationale of our choice.

In the following paragraphs the different steps of the genetic algorithm are described.

1) Encoding: The Cover Sets Scheduling Problem (CSSP) is considered as the scheduling of $K_{o p t} \alpha$-cover sets and the search space corresponds to the $K_{o p t}$ ! possible ordering of these cover sets. A solution of this ordering problem, called a chromosome in the GA, can be naturally represented by an ordered sequence $(O S)$ of the $K_{o p t} \alpha$-cover sets where each gene corresponds to the index of an $\alpha$-cover set as outlined in Figure 2.

$$
\mathrm{OS}=\left\{\mathrm{C}_{1}, \mathrm{C}_{3}, \mathrm{C}_{5}, \mathrm{C}_{4}, \mathrm{C}_{2}\right\} \leftrightarrow \begin{array}{|l|l|l|l|l|}
\hline 1 & 3 & 5 & 4 & 2 \\
\hline
\end{array}
$$

Fig. 2: Representation of a solution as an ordered sequence.

2) Fitness function: It evaluates the quality of a solution according to the first and second criteria presented in the previous sections. Assigning a score to a solution allows its comparison to other solutions. If two solutions have the same minimum distance between the uncovered periods for all the targets, the average coefficient of variation for both solutions are compared and the one with the lowest average coefficient of variation has a better uncovered periods dispersion rate. Therefore, the fitness function returns two values for a given $O S:$ i) the minimum distance between the uncovered periods and ii) the average coefficient of variation for all targets.

3) Crossover operator: Among several types of crossover operators, the LOX (Linear Ordering Crossover) [19] was adopted because it has been shown in [20] that it is well adapted for linear permutation problems. The operator LOX works as follows:

- Two crossover points are selected randomly.

- At the parents' level, the sub-sequences between the two crossover points are transferred to the children.

- Starting from the beginning of a chromosome, the genes are copied in the order in which they appear in the other parent by omitting the repeated genes.

Figure 3 shows an example of applying the crossover operator on two parent to generate two new children solutions.

4) Mutation operator: The mutation operator consists of modifying one or more genes of a solution to improve its fitness. The swap mutation operator which consists in selecting two genes to swap them was adopted. Instead of randomly selecting the two genes to swap, the implemented operator selects, as the first gene, one of the cover sets that gives the smallest distance between the uncovered periods. A search method is then used to discover which other cover set would give the best improvement when swapped with the first selected gene. Figure 4 shows an example of applying the mutation operator on one parent to generate a new child solution. In the example, target $t_{1}$ has the least dispersed uncovered periods due to three successive uncovered periods

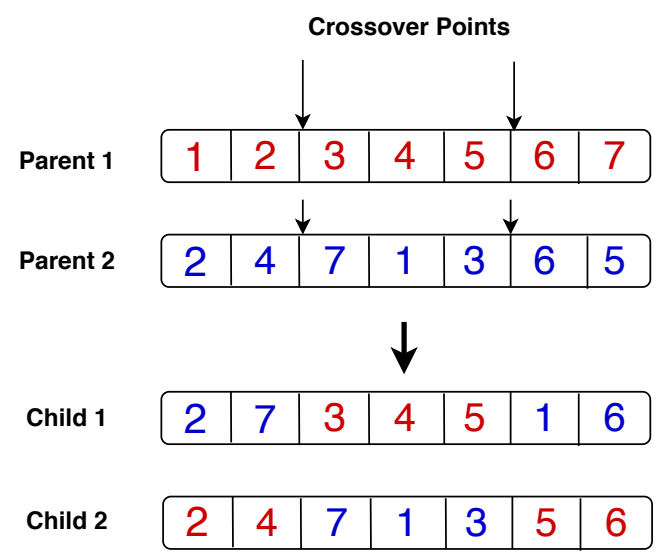

Fig. 3: The crossover operator applied on two individuals to generate two new solutions.

in cover sets $C_{4}, C_{5}$ and $C_{6}$. To increase the distance between successive uncovered periods, it is obvious that $C_{5}$ should be swapped with a cover set that does cover target $t_{1}$. Therefore, all the possible swaps are evaluated and as seen in the figure, $C_{8}$ gives the highest distance when swapped with $C_{5}$. Therefore, the mutation operator swaps these two cover sets and generates a new solution with a better dispersion rate of uncovered periods.

Figure 5 illustrates the flow chart of the proposed genetic approach to obtain good solutions for the cover sets scheduling problem. At the beginning of the algorithm, a set of solutions, represented by chromosomes and known as the initial population, is generated and the fitness of each solution is computed. At each generation of the genetic algorithm, the individuals in the population with the best fitness values are selected. The crossover and mutation operators are then applied on the selected individuals to generate new solutions. At the end of a generation, the new solutions are added to the population and in order to keep the size of the population fixed, the worst individuals in the population are removed. The iterative process is stopped when the maximum number of generations is reached.

The results of the experiments evaluating the performance of this genetic algorithm are presented in Section V-C1.

\section{EXPERIMENTS AND RESULTS}

In this section, we present the experiments conducted to assess the performance of our proposals. As mentionned in the introduction, two main optimization objectives are considered, namely: i) the network's lifetime optimization and the cover sets scheduling. The former seeks to solve the $\alpha$-coverage problem to optimality by proposing a novel BILP mathematical model, whereas the later focuses on the suitable planning way of the set covers obatained by the BILP's solver to smooth fairly the cumulated targets' uncovered time periods during the network's service. In subsection V-A, the results of solving to optimality many instances of the $\alpha$-MLP are presented. In these experiments, we evaluate the effects of considering the 


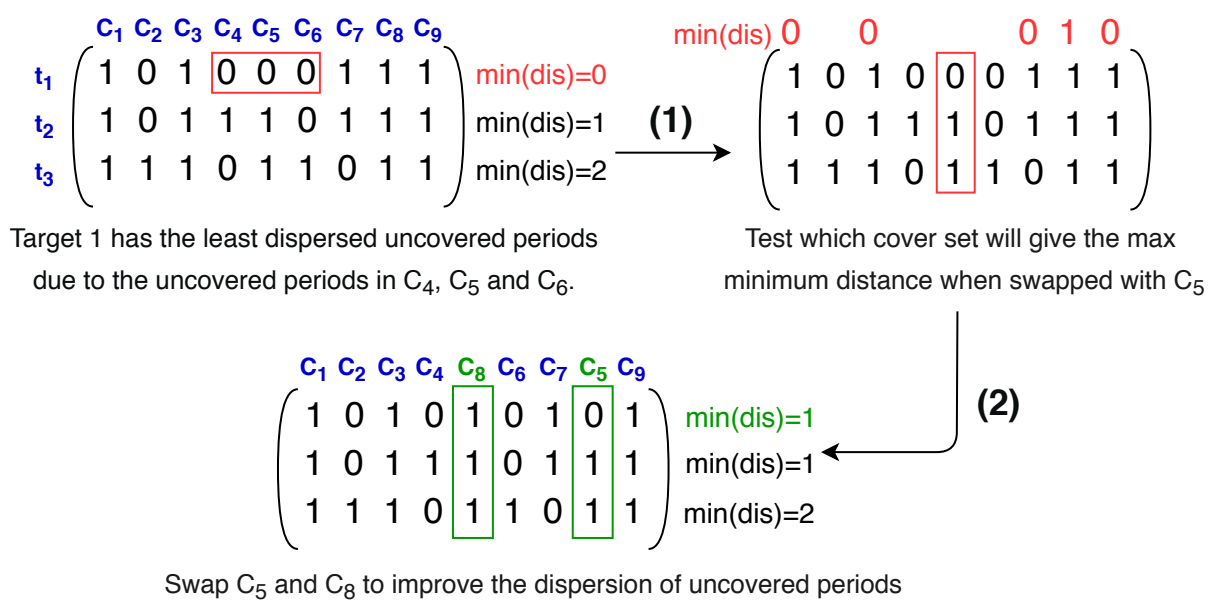

Fig. 4: The mutation operator applied on one individual to generate a better solution.

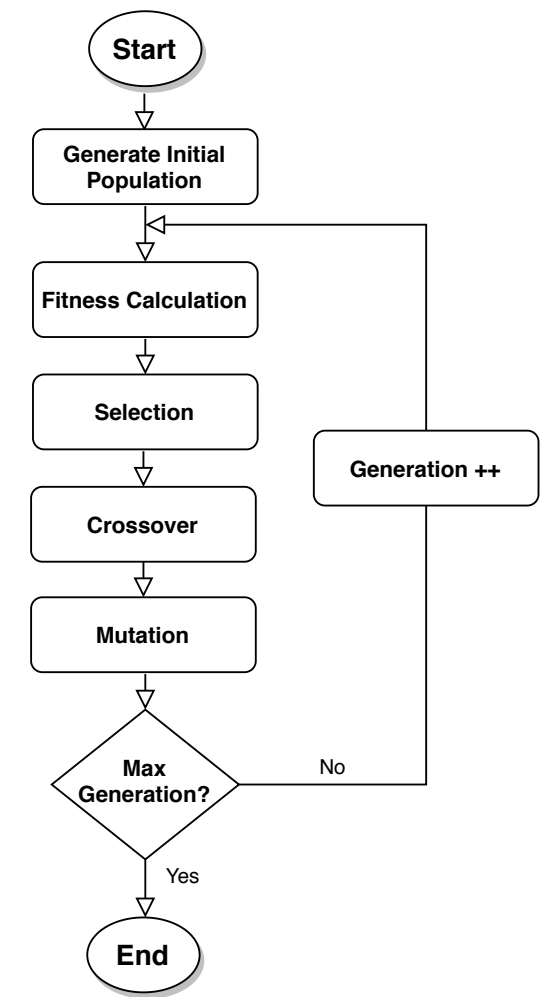

Fig. 5: General flow chart of the proposed genetic approach.

$\beta$ constraint instead of the $w_{\min }$ constraint, on the obtained network lifetime and target's coverage ratio. We also assess the quality of the upper bound of the number of $\alpha$-cover sets by comparing it for many instances with the numbers of $\alpha$-cover sets in the optimal solutions. Finally the results obtained by our method are compared to those obtained by an existing 3-steps method proposed in [7]. In subsection $\mathrm{V}-\mathrm{C}$, we compare the results obtained by default from solving the BILP to the ones provided by the proposed Genetic Algorithm and demonstrate that this GA can improve the quality of the solutions for the Cover Set Scheduling Problem. All these experiments were coded in JAVA and executed over an Intel(R) i7-8650U processor with $16 \mathrm{~GB}$ of RAM. Note that the experimental set up and the parameters used in our study are chosen in such a way that they are representative and are in line with those used in the literature (see for example [4], [5] and [6]).

\section{A. Results for $\alpha-M L P$}

IBM ILOG CPLEX 12.5 was used to solve the considered instances of the BILP, presented in Section III-B. All these instances consisted of networks with 15 targets and 10 to 40 sensors. In each instance, the $n$ sensors and $m$ targets were randomly deployed in a $500 \times 500 \mathrm{sqm}$ two-dimensional area. Each target was at least covered by $n / 4$ sensors. All the deployed sensors could communicate directly with the base station and had the same $300 \mathrm{~m}$ sensing range. Note that the sensing range value will not affect the BILP's performances. It is a system parameter which specifies which targets are monitored by each sensor. At the start of the surveillance, they had heterogeneous initial energy, varying between 1 to 12 energy units. One unit of energy allows a sensor to stay active during one unit of time and to cover during that time all the targets in its range. All the presented experiments' results are averages of 10 randomly generated instances. Four values for the activation time $d$, equal to $2,3,4$ or 6 time units, were considered in the first set of the experiments and then it was fixed to 3 time units for the rest of them. Four values of $\alpha$ equal to $1,0.85,0.75$ and 0.5 were also considered and therefore, each partial cover set had to survey at least $T_{\alpha}=15$, 13, 11 and 8 targets respectively. All the parameters of the experiments for the $\alpha$-MLP are listed in Table I.

1) The network lifetime for different values of activation time: This first experiment was conducted to evaluate the influence of the activation time on the network lifetime, obtained by solving the BILP, while varying the value of $\alpha$. 


\begin{tabular}{|l|l|}
\hline Parameter & Description \\
\hline Area & $500 \times 500 \mathrm{sqm}$ \\
\hline Number of sensors $(n)$ & $10-40$ \\
\hline Number of targets $(m)$ & 15 \\
\hline Sensing range $\left(R_{s}\right)$ & $300 \mathrm{~m}$ \\
\hline Initial energy of sensor $\left(E_{i}\right)$ & $1-12 \mathrm{unit}$ \\
\hline Activation time for $\alpha-$ cover set $(\mathrm{d})$ & $2,3,4,6$ \\
\hline Values of $\alpha$ & $1,0.85,0.75,0.5$ \\
\hline Values of $T_{\alpha}$ & $15,13,11,8$ \\
\hline
\end{tabular}

TABLE I: Simulation Parameters for $\alpha$-MLP

The following activation times were tested: $2,3,4$ and 6 time units where sensors can at most participate to $6,4,3$ or 2 cover sets respectively.

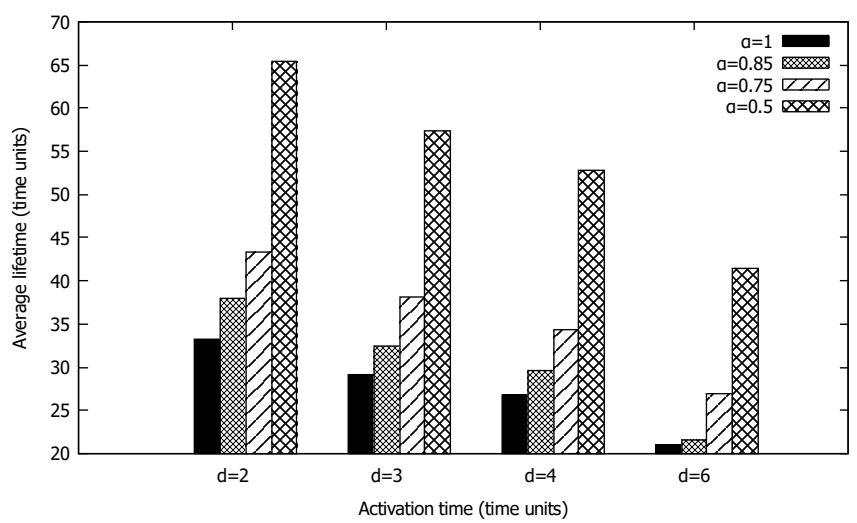

Fig. 6: Average network lifetime obtained by solving the BILP for different activation time period values under constraint $\beta$.

Figure 6 presents the average lifetime of a network composed of 20 sensors for different activation times and $\alpha$ values. As expected, with the partial coverage constraint the lifetime of the network is higher than with the complete coverage constraint. As more targets are neglected in the cover sets $(\alpha$ is decreased), the lifetime of the network increases. For example, with the activation time $d=2$, the obtained network lifetime is largely improved from $14.45 \%\left(\alpha=0.85, T_{\alpha}=13\right)$ to $96.98 \%\left(\alpha=0.5, T_{\alpha}=8\right)$ when compared to the network lifetime obtained under full coverage $(\alpha=1)$. Figure 6 also shows that the network lifetime increases when the cover set activation time is decreased. This is due to the fact that as the activation times are decreased, a sensor can participate in more $\alpha$-cover sets and can fully consume its energy, while with larger activation times, a sensor can be active in a small number of cover sets and it will waste a lot of its energy. For example, with $\alpha=0.5$, the network lifetime increases by $57.97 \%$ when considering an activation time equal to two time units $(d=2)$ instead of six time units $(d=6)$.

2) The execution time for different values of activation time: Figure 7 presents the average execution times for solving the BILP under constraint $\beta$ for different activation time values. It can be noticed that as the activation time is increased the execution time is decreased. This is due to the fact that the upper bound of the number of cover sets, $K$ is

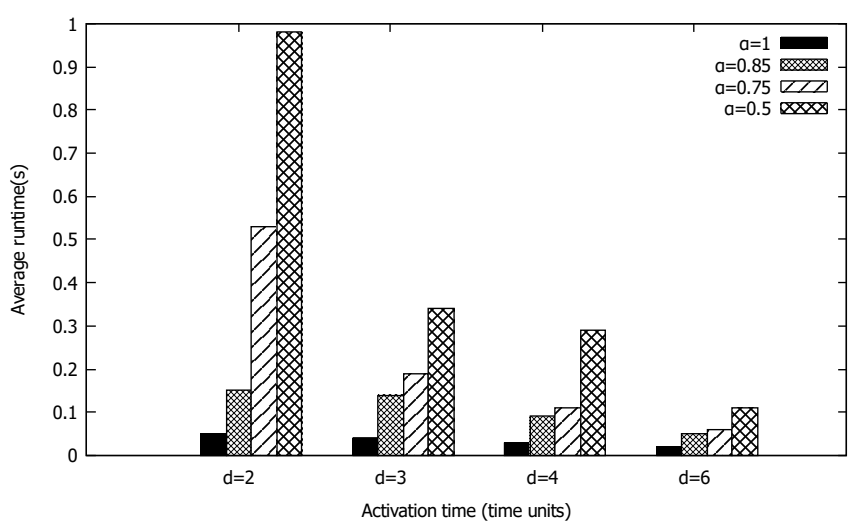

Fig. 7: Average runtime to solve the BILP for different values of activation time under constraint $\beta$.

inversely proportional to the activation time. As $K$ increases, the complexity of the BILP and the search space increases. For example, with $\alpha=0.5$, the execution time is increased by $790.9 \%$ when considering $(d=2)$ instead of $(d=6)$. Figure 7 also shows that as more targets are neglected ( $\alpha$ decreased), the execution time increases because more cover sets can be constructed and the upper bound $K$ grows which complexifies the BILP and widens the search space. For example, for $d=3$ the execution time increases by $142.85 \%$ when considering $\alpha=0.5$ instead of $\alpha=0.85$.

3) The Upper bound versus the optimal value for the number of $\alpha$-cover sets under $w_{\min }$ and $\beta$ constraint: The upper bound of the possible number of $\alpha$-cover sets $K$ computed in part III-C is used to size the BILP presented in (10). In this paragraph, we investigate whether this $K$ value is often attainable on the set of processed instances and we measure the deviation between this value and the optimal number of $\alpha$-cover sets (denoted by $K_{\text {opt }}$ ) obtained after resolution of the BILP. This value $K_{\text {opt }}$ corresponds to the number of non-zero $z_{k}$ variables in the optimal solution. We distinguish two cases, the case where the $w_{\min }$ constraint is applied, and the case where the $\beta$ constraint is applied. Table II presents the upper bound $K$ and the obtained $\alpha$-cover sets $K_{\text {opt }}$ of the BILP formulated previously under either the constraint $w_{\min }$ or the constraint $\beta$. The activation time is fixed to 3 time units.

Table II shows that the upper bounds are higher when using the $w_{\text {min }}$ constraint instead of the $\beta$ constraint. For example, with $n=30$ and $\alpha=0.75$, the upper bound is $80.7 \%$ higher with the $w_{\min }$ constraint than with the $\beta$ constraint. This is due to the fact that the constraint $\beta$ limits the network's lifetime according to the parameter $\beta$ and consequently the upper bound of the number of cover sets is also tighter. Moreover, it can be noticed that as less targets are covered in the cover sets ( $\alpha$ is decreased), the upper bound increases under either constraint $\beta$ or constraint $w_{\min }$. For example, with $n=20$, the upper bound under constraint $\beta$ increases by $73.57 \%$ when considering $\alpha=0.5$ instead of $\alpha=0.85$. Nevertheless, the quality of the obtained upper bounds considerably reduces the 


\begin{tabular}{|c|c|c|c|c|c|c|c|c|c|c|c|c|c|c|c|c|}
\hline \multirow[b]{3}{*}{$\alpha$} & \multicolumn{4}{|c|}{$\mathrm{n}=10$} & \multicolumn{4}{|c|}{$\mathrm{n}=20$} & \multicolumn{4}{|c|}{$\mathrm{n}=30$} & \multicolumn{4}{|c|}{$\mathrm{n}=40$} \\
\hline & \multicolumn{2}{|c|}{$w_{\min }$} & \multicolumn{2}{|c|}{$\beta$} & \multicolumn{2}{|c|}{$w_{\min }$} & \multicolumn{2}{|c|}{$\beta$} & \multicolumn{2}{|c|}{$w_{\min }$} & \multicolumn{2}{|c|}{$\beta$} & \multicolumn{2}{|c|}{$w_{\min }$} & \multicolumn{2}{|c|}{$\beta$} \\
\hline & $\mathrm{K}$ & $K_{o p t}$ & $\mathrm{~K}$ & $K_{o p t}$ & $\mathrm{~K}$ & $K_{o p t}$ & $\mathrm{~K}$ & $K_{\text {opt }}$ & $\mathrm{K}$ & $K_{\text {opt }}$ & $\mathrm{K}$ & $K_{o p t}$ & $\mathrm{~K}$ & $K_{\text {opt }}$ & K & $K_{o p t}$ \\
\hline 1 & 4.9 & 4.0 & 4.9 & 4.0 & 11.9 & 9.7 & 11.9 & 9.7 & 23.3 & 19.7 & 23.3 & 19.7 & 26.2 & 21.7 & 26.2 & 21.7 \\
\hline 0.85 & 12.0 & 8.8 & 5.5 & 4.2 & 25.9 & 16.6 & 14.0 & 10.8 & 44.5 & 30.7 & 27.2 & 22.5 & 54.4 & 37.3 & 30.6 & 25.1 \\
\hline 0.75 & 17.1 & 10.5 & 6.5 & 4.9 & 34.2 & 22.1 & 16.0 & 12.7 & 56.2 & 36.5 & 31.1 & 25.9 & 70.1 & 47.7 & 34.8 & 28.6 \\
\hline 0.5 & 24.6 & 14.2 & 9.9 & 8.0 & 48.3 & 29.0 & 24.3 & 19.1 & 78.1 & 46.2 & 46.9 & 38.9 & 98.5 & - & 52.5 & 43.3 \\
\hline
\end{tabular}

TABLE II: The upper bound $K$ and the obtained $\alpha$-cover sets $K_{\text {opt }}$ for different networks under either $w_{\min }$ constraint or $\beta$ constraint

number of variables and constraints in the BILP and allows us to solve to optimality larger instances than before.

4) $w_{\text {min }}$ constraint versus $\beta$ constraint: Table III presents the execution time and lifetime of the BILP formulated previously under either the constraint $w_{\min }$ or the constraint $\beta$ for the same instances as those presented in table II. As under the $\beta$ constraint and for the same reasons, when partial coverage is considered instead of complete coverage, the network lifetime also increases under the $w_{\min }$ constraint. For example, Table III shows that for the instance with $n=30$, the average network lifetime under constraint $w_{\text {min }}$ significantly improved from $55.83 \%$ with $\alpha=0.85$ to $134.51 \%$ with $\alpha=0.5$ when compared to the network lifetime under full coverage $(\alpha=1)$. Moreover, it can be noticed that for some instances considering constraint $\beta$ instead of constraint $w_{\text {min }}$ might decrease the network lifetime. For example, for the instances with $n=30$ and $\alpha=0.75$, when constraint $\beta$ is considered instead of constraint $w_{\min }$, the average network lifetime decreased by $29.04 \%$. This decrease in lifetime under the $\beta$ constraint was expected because contrary to the $w_{\min }$ constraint, it imposes a minimum coverage level per target which makes it more appropriate for real-life applications requirements. On the other hand, Table III shows that the execution times are higher when using constraint $w_{\text {min }}$ instead of constraint $\beta$. For example, with $n=40$ and $\alpha=0.75$, the execution time is $120,516 \%$ higher with $w_{\min }$ instead of constraint $\beta$. This is due to the fact that the upper bound of the number of cover sets $K$ is smaller under the $\beta$ constraint than under the $w_{\text {min }}$ constraint and the complexity of the BILP is directly related to the value of $K$. Finally, with either constraints, $w_{\min }$ or $\beta$, only the optimal solutions of small networks can be computed in a reasonable time because it is an NP-hard problem. The results of instances with $n=40$ and $\alpha=0.5$ under constraint $w_{\text {min }}$ are not displayed in Table III because they could not be solved in a reasonable time.

After comparing the effects of considering $\beta$ constraint instead of the $w_{\min }$ constraint in terms of execution time and network lifetime, in this paragraph, we compare their influence on the target's coverage percentage over the total lifetime of the network. In these experiments, the considered instances have 20 sensors and the activation time of the cover sets is fixed to 3 time units. For the sake of simplicity, all targets have the same monitoring ratio $\beta$ which is equal to $\alpha$. Figures 8,9 and 10 show for $\alpha$ equals to $0.85,0.75$ and 0.5 respectively, the percentage of coverage for each target over the total lifetime of the network under either $\beta$ constraint or $w_{\min }$ constraint. The results reveal that under the $\beta$ constraint each target is on average covered for a period equal or superior to the one under the $w_{\min }$ constraint.

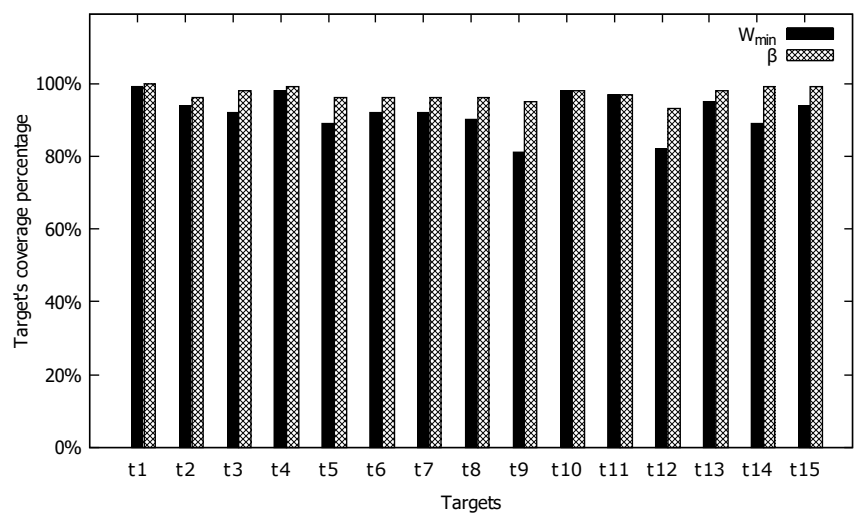

Fig. 8: The target's coverage percentage over the total lifetime of the network for $\alpha-M L P$ under $\beta$ constraint , $w_{\text {min }}$ constraint with $\alpha=0.85, n=20, \beta=\alpha=0.85$

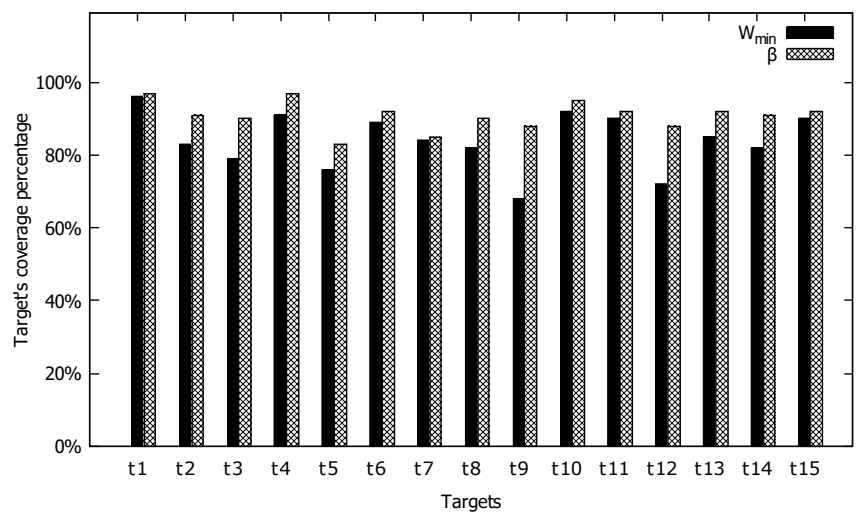

Fig. 9: The target's coverage percentage over the total lifetime of the network for $\alpha-M L P$ under $\beta$ constraint , $w_{\min }$ constraint with $\alpha=0.75, n=20, \beta=\alpha=0.75$

To prove that the $w_{\min }$ constraint is not sufficient to impose an appropriate global covering for each target, we have solved 10 instances of the $\alpha-M L P$ under the $w_{\min }$ constraint and counted the number of targets that were not covered appropriately. A target is considered as not being covered properly, if its coverage ratio is less than $\alpha$. The activation 


\begin{tabular}{|c|c|c|c|c|c|c|c|c|c|c|c|c|c|c|c|c|c|}
\hline & \multicolumn{4}{|c|}{$n=10$} & \multicolumn{4}{|c|}{$n=20$} & \multicolumn{4}{|c|}{$n=30$} & \multicolumn{4}{|c|}{$n=40$} \\
\hline & & \multicolumn{2}{|c|}{$w_{\min }$} & \multicolumn{2}{|c|}{$\beta$} & \multicolumn{2}{|c|}{$w_{\min }$} & \multicolumn{2}{|c|}{$\beta$} & \multicolumn{2}{|c|}{$w_{\min }$} & \multicolumn{2}{|c|}{$\beta$} & \multicolumn{2}{|c|}{$w_{\min }$} & \multicolumn{2}{|c|}{$\beta$} \\
\hline$\alpha$ & $T_{\alpha}$ & $\mathrm{L}$ & $\mathrm{T}(\mathrm{s})$ & $\mathrm{L}$ & $\mathrm{T}(\mathrm{s})$ & $\mathrm{L}$ & $\mathrm{T}(\mathrm{s})$ & $\mathrm{L}$ & $\mathrm{T}(\mathrm{s})$ & $\mathrm{L}$ & $\mathrm{T}(\mathrm{s})$ & $\mathrm{L}$ & $\mathrm{T}(\mathrm{s})$ & $\mathrm{L}$ & $\mathrm{T}(\mathrm{s})$ & $\mathrm{L}$ & $\mathrm{T}(\mathrm{s})$ \\
\hline 1 & 15 & 12 & 0.017 & 12 & 0.017 & 29.1 & 0.042 & 29.1 & 0.042 & 59.1 & 0.084 & 59.1 & 0.084 & 65.1 & 0.13 & 65.1 & 0.13 \\
\hline 0.85 & 13 & 26.4 & 0.05 & 12.6 & 0.02 & 49.8 & 1.38 & 32.4 & 0.14 & 92.1 & 49.78 & 67.5 & 0.49 & 111.9 & 157.79 & 75.3 & 0.78 \\
\hline 0.75 & 11 & 31.5 & 0.01 & 14.7 & 0.02 & 66.3 & 103.21 & 38.1 & 0.18 & 109.5 & 973.53 & 77.7 & 0.77 & 143.1 & 1399.15 & 85.8 & 1.16 \\
\hline 0.5 & 8 & 42.6 & 0.24 & 24 & 0.04 & 87 & 6.95 & 57.3 & 0.35 & 138.6 & 305.27 & 116.7 & 29.43 & - & - & 129.9 & 615.24 \\
\hline
\end{tabular}

TABLE III: The lifetime and execution time for different networks under either $w_{\text {min }}$ constraint or $\beta$ constraint

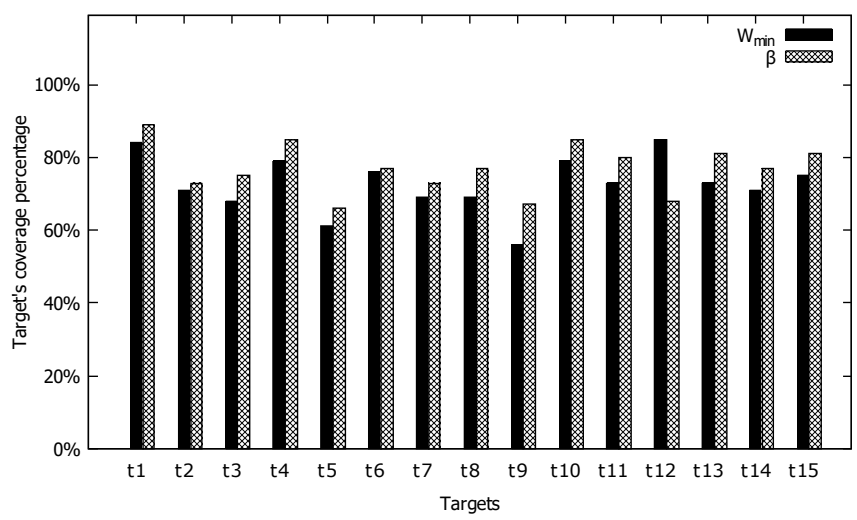

Fig. 10: The target's coverage percentage over the total lifetime of the network for $\alpha-M L P$ under $\beta$ constraint, $w_{\text {min }}$ constraint with $\alpha=0.5, n=20, \beta=\alpha=0.5$

time was fixed to 3 time units and each instance had 15 targets to monitor. Table IV shows the results of this experiment and it can be noticed that a high number of targets is undercovered with the $w_{\min }$ constraint. Therefore, constraint $w_{\text {min }}$ is not sufficient to guarantee a good coverage quality for the monitored targets. For example, for $\alpha=0.75,37$ of the 150 targets (15 targets for 10 instances) were covered for periods smaller than the desired level. On the other hand, when considering the $\beta$ constraint and when setting $\beta=\alpha$, the global and local coverage levels are always satisfied.

\begin{tabular}{|l|l|l|l|}
\cline { 2 - 4 } \multicolumn{1}{c|}{} & $\alpha=0.85$ & $\alpha=0.75$ & $\alpha=0.5$ \\
\hline Constraint $w_{\min }$ & 29 & 37 & 22 \\
\hline
\end{tabular}

TABLE IV: Number of targets in 10 instances with a coverage rate inferior to $\alpha$ under the $w_{\min }$ constraint

Moreover, for the same experiment, Table V presents the target's minimum coverage ratio under either the $w_{\text {min }}$ constraint or the $\beta$ constraint. The results show that when only considering the $w_{\min }$ constraint, the target's minimum coverage ratio is very low which means that some targets are extremely under-covered during the network's lifetime. For example, for $\alpha=0.5$, the experiment showed that at least one target is just covered during $15 \%$ of the network's lifetime. On the other hand, under the $\beta$ constraint, each target is at least covered during $\beta \times 100 \%$ of the network's lifetime. For example, replacing in the experiment constraint $w_{\min }$ by constraint $\beta$, improves the target's minimum coverage rate from 0.2 to 0.75 for $\alpha=\beta=0.75$.

\begin{tabular}{|l|l|l|l|}
\cline { 2 - 4 } \multicolumn{1}{c|}{} & $\alpha=\beta=0.85$ & $\alpha=\beta=0.75$ & $\alpha=\beta=0.5$ \\
\hline Constraint $w_{\text {min }}$ & 0.27 & 0.2 & 0.15 \\
\hline Constraint $\beta$ & 0.85 & 0.75 & 0.5 \\
\hline
\end{tabular}

TABLE V: Target's minimum coverage ratio using constraint $w_{\text {min }}$ or constraint $\beta$

5) The relative target's coverage gain under constraint $\beta$ : This section presents for each target how much its coverage would increase if the partial coverage mode under the $\beta$ constraint is adopted instead of the complete coverage mode. The relative coverage gain per target was computed as follows:

$$
\frac{\sum_{k=1}^{K}\left(y_{j, k} \times d\right)-w_{\min }}{w_{\min }} \times 100
$$

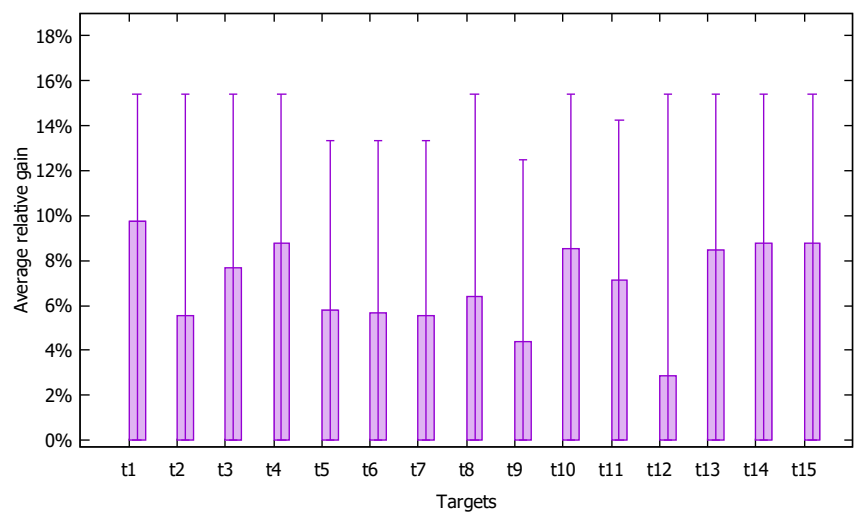

Fig. 11: The average coverage relative gain for each target under partial coverage and with $\alpha=\beta=0.85$.

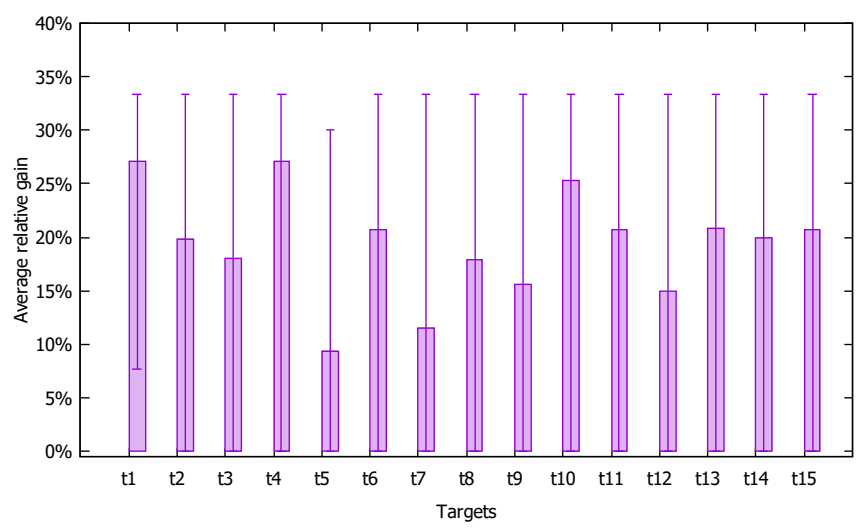

Fig. 12: The average coverage relative gain for each target under partial coverage and with $\alpha=\beta=0.75$. 


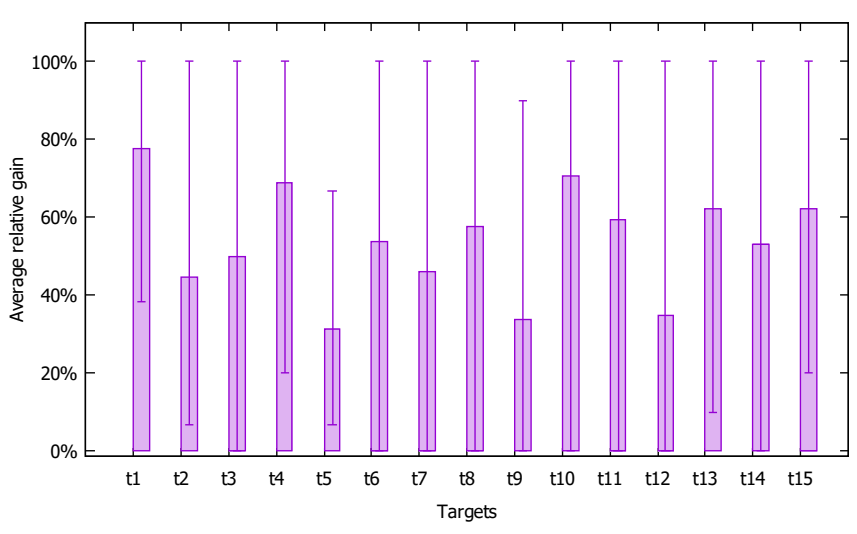

Fig. 13: The average coverage relative gain for each target under partial coverage and with $\alpha=\beta=0.5$.

Figures 11, 12 and 13 present, for each target, the average coverage relative gain under partial coverage and for $\alpha$ equal to $0.85,0.75$ and 0.5 respectively. They also display the $100 \%$ confidence interval. In this experiment, the number of sensors was fixed to 20 and the activation time for each cover set was also set to 3 time units. These figures show that with partial coverage and under the $\beta$ constraint each target is at least covered for a time period equal or bigger than the network's lifetime under the complete coverage constraint because all the coverage gain values are null or positive. On average the target's coverage is improved by $6.94 \%, 19.3 \%$ and $53.79 \%$ for $\alpha=0.85,0.75$ and 0.5 respectively. Therefore, the $\beta$ constraint can advantageously replace the $w_{\min }$ constraint because it imposes for each target a coverage level equal or superior to the one required by the $w_{\min }$ constraint without computing $w_{\min }$.

\section{B. Performance comparison between our all-in-one method and the 3-steps method}

In Table VI, we compare the performance of our allin-one method with the 3-steps method, proposed in [7]. We applied both methods on smaller instances than in the previous experiments because the 3-steps method took too much time to solve to optimality larger instances including 30 or more sensors. The comparison results show that our method outperforms the 3-steps method in all the tested instances besides the very small ones. For example, with $n=25$ and $\alpha=0.5$, the execution time of our method is on average $99.52 \%$ lower than the 3-steps method's execution time for the 10 tested instances. This is due to the time complexity of the two first steps of the 3-steps method where all the valid and elementary cover sets are enumerated. The number of possible cover sets is equal to $2^{n}-1$ which is an exponential function of $n$ and each time the number of sensors is increased by 1 , the number of possible cover sets doubles. For this reason, the 3 -steps method cannot solve in a reasonable time an instance including more than 25 sensors. It took around four hours to solve an instance with $n=30$ and $\alpha=1$.
Moreover, as $\alpha$ decreases in the partial coverage case, the number of the enumerated valid and elementary $\alpha$-cover sets, $L$, increases. For each valid and elementary $\alpha$-cover set, a constraint is added to the linear model in the third step of the 3-steps method. Therefore, as $L$ increases the model takes more memory and becomes harder to solve by the IBM ILOG CPLEX which imposes a size limit on the model. On the other hand, our method can compute the optimal solution for larger instances, up to $n=40$, with an execution time inferior to 10 minutes as shown in Table III.

In conclusion, our approach outperforms the 3-steps method and can solve larger instances.

\section{Results for CSSP}

In this section, we evaluate the proposed genetic algorithm to optimize the scheduling of the cover sets of the solutions obtained by the resolution of the BILP. The crossover and the mutation rates of the GA were set to $80 \%$ and $20 \%$ respectively. As described in Section IV-C, a chromosome represents the order of the $\alpha$-cover sets in a given solution and its size is always equal to $K_{o p t}$. All the experiments' results are averages for 10 randomly generated instances. All the GA's parameters are listed in Table VII.

\begin{tabular}{|l|l|}
\hline Parameter & Description \\
\hline Number of generations & 100 \\
\hline Population size & 100 \\
\hline Probability of crossover & 0.8 \\
\hline Probability of mutation & 0.2 \\
\hline
\end{tabular}

TABLE VII: The genetic algorithm's parameters

1) The GA versus the exhaustive search method for Cover Sets Scheduling Problem on small networks: Solving the cover sets scheduling problem seeks to plan efficiently the cover sets of a given solution in order to smooth fairly the targets' uncovered periods throughout the network's lifetime. To show the usefulness of our proposal, two scheduling approaches were compared in this section: the exhaustive (brute-force) search method and the proposed GA. Their results were also compared to the default scheduling obtained by solving the BILP. Due to the factorial time complexity of the brute-force search, this method can only be applied to small instances and therefore the experiments of this section are limited to solutions including 6 to 11 partial cover sets.

\begin{tabular}{|c|c|c|c|c|c|c|}
\cline { 2 - 7 } \multicolumn{1}{c|}{} & \multicolumn{2}{c|}{ Default scheduling } & \multicolumn{2}{c|}{ Exhaustive search method } & \multicolumn{2}{c|}{ GA } \\
\hline$K_{\text {opt }}$ & min(dis) & Average CV & min(dis) & Average CV & $\min ($ dis $)$ & Average CV \\
\hline 6 & 0.3 & 0.63 & 0.9 & 0.32 & 0.9 & 0.32 \\
\hline 7 & 0 & 1.56 & 0.5 & 0.62 & 0.4 & 0.64 \\
\hline 8 & 0.3 & 1.27 & 0.9 & 0.44 & 0.9 & 0.44 \\
\hline 9 & 0 & 2.38 & 0 & 1.06 & 0 & 1.21 \\
\hline 10 & 0.1 & 0.6 & 1.9 & 0.15 & 1.8 & 0.17 \\
\hline 11 & 0.5 & 0.39 & 2.7 & 0.06 & 2.6 & 0.07 \\
\hline
\end{tabular}

TABLE VIII: Minimum of the minimum distances and average coefficient of variation for cover sets scheduling returned by the BILP (default), the exhaustive search method and the GA.

In Section IV, two criteria were proposed to compare the solutions returned by the search methods: p-dispersion and 


\begin{tabular}{|c|c|c|c|c|c|c|c|c|c|c|c|c|c|}
\cline { 2 - 14 } \multicolumn{2}{c|}{} & \multicolumn{4}{c|}{$\mathrm{n}=10$} & \multicolumn{4}{c|}{$\mathrm{n}=15$} & \multicolumn{2}{c|}{$\mathrm{n}=20$} & \multicolumn{3}{c|}{$\mathrm{n}=25$} \\
\hline$\alpha$ & $T_{\alpha}$ & $\mathrm{L}$ & $\begin{array}{c}\text { Rt } \\
\text { (3-steps } \\
\text { method) }\end{array}$ & $\begin{array}{c}\text { Rt } \\
\text { (all-in-one } \\
\text { method) }\end{array}$ & L & $\begin{array}{c}\text { Rt } \\
\text { (3-steps } \\
\text { method) }\end{array}$ & $\begin{array}{c}\text { Rt } \\
\text { (all-in-one } \\
\text { method) }\end{array}$ & L & $\begin{array}{c}\text { Rt } \\
\text { (3-steps } \\
\text { method) }\end{array}$ & $\begin{array}{c}\text { Rt } \\
\text { (all-in-one } \\
\text { method) }\end{array}$ & $\begin{array}{c}\text { Lt } \\
\text { (3-steps } \\
\text { method) }\end{array}$ & $\begin{array}{c}\text { Rt } \\
\text { (all-in-one } \\
\text { method) }\end{array}$ \\
\hline 1 & 15 & 12 & 0.034 & 0.042 & 22.5 & 0.383 & 0.036 & 29.1 & 9.062 & 0.084 & 46.8 & 378.145 & 0.059 \\
\hline 0.85 & 13 & 12.6 & 0.039 & 0.02 & 24.9 & 0.476 & 0.064 & 32.4 & 8.92 & 0.14 & 53.4 & 499.964 & 0.301 \\
\hline 0.75 & 11 & 14.7 & 0.078 & 0.02 & 29.1 & 0.417 & 0.084 & 38.1 & 9.55 & 0.18 & 61.2 & 521.282 & 0.394 \\
\hline 0.5 & 8 & 24 & 0.046 & 0.04 & 44.7 & 0.463 & 0.139 & 57.3 & 9.72 & 0.35 & 91.5 & 408.854 & 1.957 \\
\hline
\end{tabular}

TABLE VI: Comparison of the two methods in terms of running time (Rt) in seconds.

coefficient of variation. Table VIII presents the minimum of the minimum distances between uncovered periods $(\min (\operatorname{dis}))$ and the coefficient of variation of these distances $(\mathrm{CV})$ for the best solutions found by each of the three methods with the number of cover sets varying from 6 to 11 . It can be noticed that as expected the exhaustive search method always returns the solutions with highest $\min (d i s)$ and $\mathrm{CV}$, which are the best solutions according to the chosen criteria. It can also be seen that the $\min (d i s)$ and $\mathrm{CV}$ of the solutions returned by the GA are very close to the ones returned by the exhaustive search method. For some instances, like when $K_{\text {opt }}$ is equal to 6 or 8 , the GA finds the optimal scheduling for the cover sets. For the other instances, the difference between the $\min (d i s)$ of the optimal solution and the one returned by the GA is less than or equal to 0.1. In all the instances, the GA improves the default scheduling returned by the BILP. Table VIII also shows that in some cases, as with $K_{o p t}=9$, the $\min$ (dis) criterion is not sufficient to compare the obtained solutions and the second criterion, $\mathrm{CV}$, must be considered. As a consequence, the results in Table VIII highlight that if the obtained $\alpha$-cover sets are scheduled a a suitable way, we can achieve in a reasonable time a well-balanced smoothing of the targets' uncovered periods throughout the network's lifetime.

2) The performance of the proposed GA on large networks: In order to evaluate the performance of the proposed GA on large networks and since the exhaustive search cannot solve them in a reasonable time, the scheduling returned by the GA was only compared to the default scheduling. The GA's parameters, crossover and mutation rates, and initial population size, were kept the same as in the previous experiments. On the other hand, the number of partial cover sets to schedule varied between 25 and 150 . When the ratio of the maximum number of uncovered periods per target to the number of periods is high, most of the solutions give the same minimum of the minimum distances, the first criterion is not sufficiently discriminatory. For this reason, we only focus on the second criterion in this section. Hence, Figure 14 only presents the average coefficient of variation of the solutions returned by default or by the GA for different numbers of $\alpha$-cover sets. It can be noticed that the scheduling returned by the GA is better than the one returned by default for all the considered configurations. The improvement over the default scheduling varies from one instance to the other and it is hard to quantify this improvement because it also depends on the quality of the default scheduling. For example, the obtained improvement is equal to $49.05 \%$ for $K_{o p t}=75$ where the default scheduling is probably very poor and there is a lot of room for improvement. On the other hand, for the 150 cover sets case, the GA does not significantly improve over the default scheduling which is already of good quality.

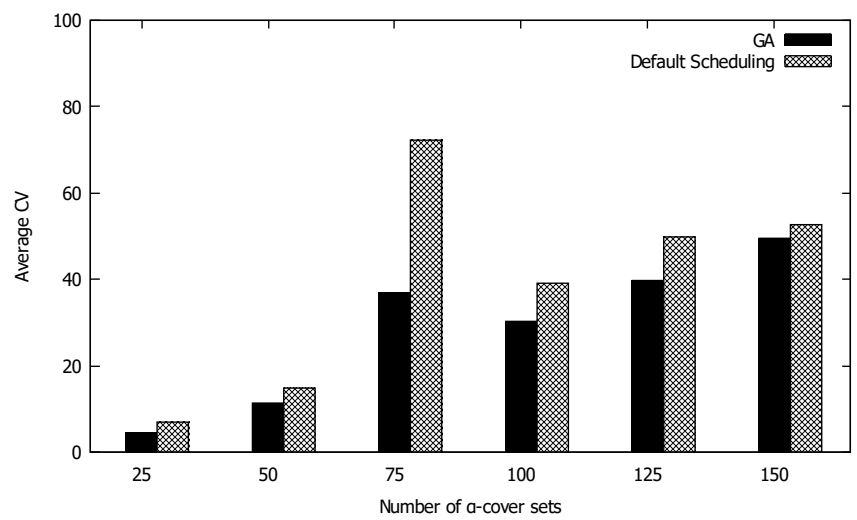

Fig. 14: Average coefficient of variation for solutions of different problem sizes returned by the BILP (default) or the GA.

\section{CONCLUSIONS}

In this paper, we have addressed the problem of partial coverage in heterogeneous sensor networks. The aim is to organize the sensor nodes into a number of non-disjoint subsets nodes that are scheduled successively to improve the network's QoS under the constraints of energy saving and partial coverage. To this end, a novel mathematical BILP is proposed to solve to optimality the $\alpha$-coverage problem. Moreover, provable guarantees of the upper bound for the number of cover sets that can be built are given. Unlike earlier works in the literature, to improve the coverage quality of the network while prolonging its lifetime, we provided necessary and sufficient condition constraints to meet, at the same time, both global and local monitoring quality thresholds. Another important contribution of this paper is the design of an efficient cover sets scheduling to fairly smooth the targets' uncovered periods during the lifetime of the network. Different scenarios were studied and the obtained results corroborate the merits of our proposals.

Future studies should target the case of large scale networks where nodes have to decide cooperatively and in a distributed way which of them will remain in sleep or active mode while at least ensuring the minimum level of coverage quality. In this 
context, particular attention should be paid for connectivity between senors presenting critical articulation points in the network. We expect a difficult challenging trade-off between the induced communication costs, the network's energy consumption, and the achieved coverage quality.

\section{REFERENCES}

[1] Y. Wu, C. Ai, S. Gao, and Y. Li, "P-percent coverage in wireless sensor networks," in International Conference on Wireless Algorithms, Systems, and Applications. Springer, 2008, pp. 200-211.

[2] M. Gentili and A. Raiconi, " $\alpha$-coverage to extend network lifetime on wireless sensor networks," Optimization Letters, vol. 7, no. 1, pp. 157172, 2013.

[3] D. Zorbas, D. Glynos, P. Kotzanikolaou, and C. Douligeris, "Solving coverage problems in wireless sensor networks using cover sets," Ad Hoc Networks, vol. 8, no. 4, pp. $400-415,2010$.

[4] F. Carrabs, R. Cerulli, A. Raiconi et al., "A hybrid exact approach for maximizing lifetime in sensor networks with complete and partial coverage constraints," Journal of Network and Computer Applications, vol. 58 , pp. 12-22, 2015.

[5] S. Chand, B. Kumar et al., "Selective $\alpha$-coverage based heuristic in wireless sensor networks," Wireless Personal Communications, vol. 97, no. 1, pp. 1623-1636, 2017.

[6] S. Das and M. K. Debbarma, "A survey on coverage problems in wireless sensor network based on monitored region," in Advances in Data and Information Sciences. Springer, 2019, pp. 349-359.

[7] Y. E. Ahmed, K. H. Adjallah, R. Stock, I. Kacem, and S. F. Babiker, "Ndsc based methods for maximizing the lifespan of randomly deployed wireless sensor networks for infrastructures monitoring," Computers \& Industrial Engineering, vol. 115, pp. $17-25,2018$.

[8] M. Cardei, M. T. Thai, Y. Li, and W. Wu, "Energy-efficient target coverage in wireless sensor networks," in Proceedings IEEE 24th Annual Joint Conference of the IEEE Computer and Communications Societies. vol. 3. IEEE, 2005, pp. 1976-1984.

[9] F. Castaño, E. Bourreau, N. Velasco, A. Rossi, and M. Sevaux, "Exact approaches for lifetime maximization in connectivity constrained wireless multi-role sensor networks," European Journal of Operational Research, vol. 241, no. 1, pp. 28-38, 2015.

[10] F. Castaño, A. Rossi, M. Sevaux, and N. Velasco, "A column generation approach to extend lifetime in wireless sensor networks with coverage and connectivity constraints," Computers \& Operations Research, vol. 52, pp. 220-230, 2014.

[11] R. Cerulli, M. Gentili, and A. Raiconi, "Maximizing lifetime and handling reliability in wireless sensor networks," Networks, vol. 64, no. 4, pp. 321-338, 2014

[12] M. Cardei, J. Wu, and M. Lu, "Improving network lifetime using sensors with adjustable sensing ranges," International Journal of Sensor Networks, vol. 1, no. 1-2, pp. 41-49, 2006.

[13] H. Mostafaei and M. S. Obaidat, "A greedy overlap-based algorithm for partial coverage of heterogeneous wsns," in GLOBECOM 2017-2017 IEEE Global Communications Conference. IEEE, 2017, pp. 1-6.

[14] K. Deschinkel, "A column generation based heuristic to extend lifetime in wireless sensor network," Sensors and Transducers Journal, vol. Vol. 14-2, pp. 242-253, 2012, 10.1007/s10878-010-9332-8.

[15] E. Erkut, "The discrete p-dispersion problem," European Journal of Operational Research, vol. 46, no. 1, pp. 48-60, 1990.

[16] A. Suzuki, R. Uehara, T. Uno, and K. Wasa, "Max-min 3-dispersion problems," in Computing and Combinatorics: 25th International Conference, COCOON 2019, Xi'an, China, July 29-31, 2019, Proceedings. Springer, p. 291

[17] S. S. Ravi, D. J. Rosenkrantz, and G. K. Tayi, "Heuristic and special case algorithms for dispersion problems," Operations Research, vol. 42, no. 2, pp. 299-310, 1994.

[18] Y. Okamoto, Y. Otachi, T. Saitoh, R. Uehara, T. Uno, and K. Wasa, "Exact algorithms for the max-min dispersion problem," in Frontiers in Algorithmics: 12th International Workshop, FAW 2018, Guangzhou, China, May 8-10, 2018, Proceedings, vol. 10823. Springer, 2018, p. 263.

[19] J. H. Holland, Adaptation in Natural and Artificial Systems. Ann Arbor, MI: University of Michigan Press, 1975, second edition, 1992.
[20] P. Lacomme, C. Prins, and W. Ramdane Cherif, "Evolutionary algorithms for periodic arc routing problems," European Journal of Operational Research, vol. 165, pp. 535-553, 092005. 